\title{
Application of Ant Colony Neural Network to Credit Evaluation of Small and Middle Enterprises
}

\author{
HongJing Liu \\ Department of Economics, Qinhuangdao Institute of Technology, Qinhuangdao, 066000,China
}

Keywords: Ant colony optimization algorithm; artificial neural network; credit evaluation

\begin{abstract}
In order to improve capacity of BP neural networks and make short term credit evaluation of small and middle enterprises forecasting more accurate and fast, presents a credit model-based the ACO neural network. Based on the analysis of the importance of credit and according to the demands of credit evaluation of small and middle enterprises, uses ACO algorithm to train neural network. And then this network model is applied to credit evaluation system of small and middle enterprises. Finally, using training samples and test samples,can detect the ant colony neural network. The result demonstrates that the ACO neural network has strong generalization ability than those of the traditional BP neural network method, and that application of credit evaluation system of small and middle enterprises has very high accuracy rate.
\end{abstract}

\section{Introduction}

Artificial neural network from the origin to the present, no substantive progress on the connection weights of the problems in the research on the process . Then BP neural network is put forward, and provides a method to solve this problem.In the present stage in the field of artificial neural network, the application of BP neural network is still the most extensive. But it also has some shortcomings, the training time is long, easy to fall into local minimum.

Genetic algorithm with global searching ability, can effectively avoid the problem of BP neural network into a local optimal. Using the method of genetic algorithm and BP neural network combination, try to analyze the genetic algorithm to optimize the network structure and parameters. By modifying the initial values and parameters know which genetic algorithm to a certain degree of improvement stability and high efficiency of the neural network, can be optimized for the original algorithm.

\section{Neural network}

BP (Back Propagation) network is a group of scientists led by Rumelhart and McCelland put forward in 1986, is a kind of error back-propagation algorithm for training multilayer feed-forward network, its learning rule is to use the method of steepest descent, to constantly adjust the network weight value and threshold value by back propagation, make the network and the minimum sum of square error. BP neural network is mainly composed of an input layer, one or more hidden layers and one output layer, the mutual connection between the layers of neurons, but between each layer between the neurons are not connected relationship.

The learning process of the BP neural network model composed of by two parts, forward and backward .In the forward process of communication, information from the input layer through the middle hidden layer to the output layer weighted propagation, in the output layer to obtain the input of the network response, output value by comparing the function calculation and target output value, if there are errors, error back propagation along the route before the return, i.e., from the output layer after each intermediate the hidden layer to adjust the connection weights, finally back to the output layer, to reduce the error, with the neural network error reverse spread to revise the weights continuously, the entire network accuracy of input information in response to natural also improved. 


\section{The credit evaluation index system of enterprise}

In the credit evaluation system, the research of domestic and foreign enterprises on the basis of analysis of the complementary, in accordance with the basic principles of the evaluation index system, combined with the characteristics of credit evaluation of SMEs in China or the actual situation, in this paper from executive ability $\mathrm{t} 1$, the operator performance $\mathrm{t} 2$, the scale of the enterprise $t 3$, the level of enterprise management $t 4$, the level of technical equipment of enterprises $\mathrm{t} 5$, reputation $\mathrm{t} 6$, debt paying ability $\mathrm{t}$, profitability $\mathrm{t} 8$, operation ability $\mathrm{t} 9$, the innovation input material $t 10$, the innovation of financial resources $t 11$, the innovation of the,human input $\mathrm{t} 12$, innovation effect $\mathrm{t} 13$, product sales forecasting $\mathrm{t} 14$, market forecast $\mathrm{t} 15$, construct the credit evaluation index system of small and medium sized enterprises(SME).Both benefit index and cost index,, can objectively reflect the enterprise credit.The evaluation object are not uniform metrics between each index, and to quantify the qualitative index and quantitative index standardization. The small and medium-sized enterprise credit evaluation index system as shown in table 1.

Table 1 credit evaluation index system of SME

\begin{tabular}{|c|}
\hline Indexes \\
\hline The basic quality of enterprise managers $(t 1)$ \\
\hline The operator performance $(\mathrm{t} 2)$ \\
\hline The scale of the enterprise $(\mathrm{t} 3)$ \\
\hline The level of enterprise management ( $t 4)$ \\
\hline The level of technical equipment of enterprises $(\mathrm{t} 5)$ \\
\hline Reputation（t6） \\
\hline Debt paying ability ( $\mathrm{t} 7)$ \\
\hline Profitability（t8） \\
\hline Operation ability（t9） \\
\hline The innovation input material $(\mathrm{t} 10)$ \\
\hline The innovation of financial resources $(\mathrm{t} 11)$ \\
\hline The innovation of the,human input (t12) \\
\hline Innovation effect $\quad(\mathrm{t} 13)$ \\
\hline Product sales forecasting ( $\mathrm{t} 14)$ \\
\hline Market forecast $(\mathrm{t} 15)$ \\
\hline
\end{tabular}

\section{Ant colony algorithm to optimize the neural network model}

Ant colony algorithm is a kind of bionic optimization of the emerging field of evolutionary algorithms, ant colony algorithm makes full use of the ant colony selection, update and coordination mechanism, to find the shortest path to food through the exchange of information between individuals, that is to find the optimal solution.,

Ant Colony Algorithm (ACA) is an intelligence-optimized algorithm coming from the illumination of food-seeking behavior by ants based on the shortest route of foraging. Ants can find the optimal path between nest and food through a continuous iteration and update. Ants will release some pheromone in current path when they are in the process of finding a path. If the path is longer, lower pheromone will be released. When tripping over obstacles, ants will choose a path randomly. The probability of choose a path is determined by the concentration of information on this path. The pheromone is volatile; as time goes by, the pheromone concentration on the optimal path will be getting more and more, and eventually the entire ant colony will find the optimal path.

The ant colony algorithm is typically applied in traveling salesman problem (TSP). The traveling salesman needs to go through all cities and returns to the starting city, and looks for the shortest path. In addition, each city is allowed only to pass once, except for the starting city two times 
repeated, other cities do not repeat. If $m$ ants are randomly placed into $n$ cities, each ant should comply with the following rules:

(1) Single ant will release pheromone according to the length of the path and select the next path with corresponding probability.

(2) Each ant can not select the path that has gone for the next path in this cycles,which means ants have memory function $t a b u_{k}$

(3) Update the pheromone of each path that has gone through after single ant completing a cycle. Let $\tau_{i j}(t)$ be the residual information quantity of the connection between city $i$ and city $j$ at time $t ; d_{i j}$ is the distance between the city $i$ and city $j$. The information quantity of each path are equal at initial moment. Let $\tau_{i j}(0)=c$ ( $c$ is an arbitrary constant). In the process of movement, ant $k$ decide the shifting direction through the information quantity of various path. $p_{i j}^{k}(t)$ is the ant $k$ 's transition probability between city $i$ and city $j$ at time $t$.

$$
p_{i j}^{k}(t)=\left\{\begin{array}{c}
\frac{\tau_{i j}^{\alpha} \eta_{i j}^{\beta}}{\sum_{k \in \text { allowed }_{k} \tau_{i k}^{\alpha} \eta_{i k}^{\beta}}^{\beta}}, j \in \text { allowed }_{k} \\
0 \text { others }
\end{array}\right.
$$

where allowed $_{k}=\{0,1,2, \ldots, n-1\}, \alpha, \beta$ is the role of information accumulated in different cities choose in the ant $k$ 's process of movement $\eta_{i j}=1 / d_{i j}$;

when ant complete a cycle, the pheromone will be updated according to the following formula:

$$
\tau_{i j}(t+n)=(1-\rho) \tau_{i j}(t)+\Delta \tau_{i j}(t, t+n)
$$

where $\rho$ is the volatile factor, $1-\rho$ represents residual level, $\rho \in(0,1)$;

$$
\Delta \tau_{i j}(t, t+n)=\sum_{k=1}^{n} \Delta \tau_{i j}^{k}(t, t+n)
$$

where $\Delta \tau_{i j}(t, t+n)$ is the pheromone increment between city $i$ and city $j$ from time $t$ to time $t+n, \quad \Delta \tau_{i j}(0)=0 ; \Delta \tau_{i j}^{k}(t, t+n)$ is the ant $k$ 's released pheromone between city $i$ and city $j$ from time $t$ to time $t+n$, its expression is:

$$
\begin{aligned}
& \Delta \tau_{i j}^{k}(t, t+n)= \\
& \left\{\begin{array}{l}
Q / L_{k} \text { if ant' } k^{\prime} \text { passed route 'ij' } \\
0 \quad \text { if } \text { ant' }^{\prime} k^{\prime} \text { didn't pass route' } i j^{\prime}
\end{array}\right\}
\end{aligned}
$$

where $Q$ is a constant, $L_{K}$ is the total length of the ant moved in this cycle.

When ants complete a cycle, record the optimal path, and repeat the above process until the predetermined iteration times, then come to the final optimal path.

Get the optimal solution by using ant algorithm colony to assign weights and thresholds of neural network, the weights using gradient descent method for neural network for further correction. So as to achieve the purpose of optimization of neural network.

\section{Empirical Analyses}

(1) data acquisition and evaluation standard design

Evaluation criteria shown in Table 2. 
Table 2 Evaluation criteria

\begin{tabular}{cccccc}
\hline $\begin{array}{c}\text { Evaluation } \\
\text { grade }\end{array}$ & I & II & III & IV & V \\
\hline Score interval & $\begin{array}{c}(0.8,1 \\
)\end{array}$ & $\begin{array}{c}(0.6,0.8 \\
)\end{array}$ & $(0.4,6)$ & $\begin{array}{c}(0.2,4 \\
)\end{array}$ & $\begin{array}{c}(0,0.2 \\
)\end{array}$ \\
\hline
\end{tabular}

Calculation of 500 samples are generated by a rigorous mathematical model at all levels of evaluation standard, 100 samples are generated between each of the two evaluation criteria, randomly selected 450 of them as training samples, the remaining 50 to test the neural network.

(2) The model training and results analysis

The network structure is: the number of network input layer neurons number index depends on the evaluation, is set to 15 , the number of hidden neurons is set to 10 , the output layer is 1 , namely the assessment results. Firstly, the BP algorithm is used for training, learning step is 5, the weight convergence factor is 0.0001 , the error convergence factor of 0.00001 , hidden layer and output layer activation function for the nonlinear sigmoid function. Connection number $\mathrm{m}=75$ of the network, ant colony algorithm parameters $\mathrm{Q}=0.7, \mathrm{~S}=30, \mathrm{Q},=100$. Neural network Pi parameters for the random number between -10 to $10, \mathrm{~N}=40$.

To train and test the network using continuous target, the normalized raw data,now the evaluation of network application of the trained in 7 for comments, seethe network output and the evaluation results in table 2 . If using the traditional BP algorithm to train network to achieve similar accuracy requires about 10000 times, the results show that the accuracy of the application based on Ant Colony Neural Network in credit evaluation of SMEs has the higher, faster convergence speed and network.

Table 3 To comment on the network output and the evaluation results

\begin{tabular}{cccccccc}
\hline $\begin{array}{c}\text { Evaluation } \\
\text { standard }\end{array}$ & 1 & 2 & 3 & 4 & 5 & 6 & 7 \\
\hline T1 & 0.93 & 0.96 & 0.79 & 0.58 & 0.91 & 0.62 & 0.80 \\
T2 & 0.89 & 0.98 & 0.86 & 0.60 & 0.80 & 0.98 & 0.61 \\
& & & & & & & \\
T13 & 0.70 & 0.94 & 0.75 & 0.62 & 0.72 & 0.73 & 0.66 \\
T14 & 0.62 & 0.90 & 0.68 & 0.55 & 0.73 & 0.70 & 0.60 \\
T15 & 0.78 & 0.95 & 0.77 & 0.50 & 0.78 & 0.77 & 0.68 \\
Assessment & 0.72 & 0.98 & 0.54 & 0.18 & 0.58 & 0.55 & 0.33 \\
objectives & & & & & & & \\
& 0.718 & 0.98 & 0.53 & 0.17 & 0.580 & 0.56 & 0.34 \\
Network output & 3 & 33 & 75 & 92 & 4 & 23 & 21 \\
Evaluation grade & II & I & III & V & III & III & IV \\
\hline
\end{tabular}

By the experiments (see Table 4) we can see that the ant colony neural network learning times is far less than the BP neural network, its convergence speed is faster, higher operation efficiency.

Table 4 Comparison of neural network and neural network based on ACO

\begin{tabular}{|c|c|c|c|}
\hline Algorithm & $\begin{array}{c}\text { Learning } \\
\text { times }\end{array}$ & $\begin{array}{c}\text { running time } \\
(\mathrm{s})\end{array}$ & $\begin{array}{c}\text { The correct } \\
\text { rate }\end{array}$ \\
\hline neural network & 30000 & 124 & $97.38 \%$ \\
\hline $\begin{array}{c}\text { neural network } \\
\text { based on ACO }\end{array}$ & 4200 & 60 & $97.59 \%$ \\
\hline
\end{tabular}

\section{Conclusion}

The dual advantages of artificial neural network based on ACO algorithm with ant colony algorithm and artificial neural network, the network has higher convergence rate and strong ability to learn. Examples show that, the neural network model based on ant colony algorithm has a higher 
classification capability, able to draw more fair, fair evaluation results, the evaluation model of small and medium-sized enterprises credit evaluation is feasible, and can be directly used this model to evaluate, thereby reducing the evaluation workload, reduce the subjectivity of evaluation, to improve the rationality of the evaluation results .

\section{References}

[1] Ellabib I , Calamai P, Basir O.Exchange strategies for multiple Ant Colony System[J].Information Sciences,2007,177:1248-1264.

[2] HE Jian-guo,JIANG Guo-yin,TIAN Bo. Credit evaluation system research based on improved BP neural network[J]. Computer Engineering and Design,2006, 2(4):605-607.

[3] CHEN De-yu, ZHANG Ren-zhong,SHEN Ji-hong. Study on multi-objective reactive power optimization based on genetic algorithm with adaptive weight[J]. Power System Protection and Control, 2010, 38(6): 1-7,25.

[4] CHAI Yi,YIN Hong-peng,LI Da-jie. BP neural network adaptive optimization design based on the improved genetic algorithm[J]. Chongqing university journal, 2007,30(4):91-96.

[5] LI Yong,WANG Jian-jun,CAO Li-hua. Real time optimal load dispatch of power plant based on back propagation neural network[J]. Power System Protection and Control, 2011, 39(17):87-92.

[6] GAO Xian-jun,ZHANG Jie,HONG Yu. Neural network technology research Based on genetic algorithm[J]. Equipment manufacturing technology, 2010,2(2):8-10.

[7] ZHU Wen-long.Application research of BP neural network in Multi-objective optimization based on genetic algorithm[D]. Harbin: Harbin university of technology,2009. 